\title{
DESENVOLVIMENTO DE UM SISTEMA VIRTUAL PARA TREINAMENTO MUSCULAR POR MIOFEEDBACK
}

\author{
A.N. Moraes*, E. S. Lawrence*, A.S. Nakagawa* e A.B. Soares* \\ *Universidade Federal de Uberlândia, Uberlândia, Brasil \\ e-mail: adrielle.nazar@hotmail.com
}

Resumo: Aprender a controlar diferentes tipos de próteses que substituem os membros que realizam tarefas cotidianas pode vir a ser um objetivo difícil a ser alcançado. Neste sentido, usuários de próteses mioelétricas podem ter dificuldade em determinar a ativação muscular necessária para efetuar os comandos desejados, dificultando o processo de aprendizagem. Nesse sentido, aplicações baseadas em biofeedback vêm se tornando ferramentas poderosas em processos terapêuticos que visam a autorregulação do indivíduo, tendo em vista que o treinamento contínuo é a melhor maneira de aprimorar o controle. Através do miofeedback, o usuário é capaz de compreender com maior precisão se a tarefa requerida está sendo realizada da maneira desejada ou não. Esse estudo propõe um sistema que recebe o sinal eletromiográfico (EMG) e o utiliza como sinal de referência para o controle de uma prótese virtual. O usuário recebe, por meio de feedbacks visuais, informações sobre o seu desempenho e do comportamento do sinal EMG do músculo monitorado.

Palavras-chave: miofeedback, próteses mioelétricas, EMG.

\begin{abstract}
Learning how to control different kinds of prostheses that replaces the limbs used to perform daily actives can be a difficult goal to achieve. One example is the use of myoelectric prostheses. In this case, the users might have difficulties in understanding how to properly control their muscles in order to achieve the desired commands. Thus, biofeedback based applications are becoming powerful tools in therapeutic processes to the individual's self-regulation and continuous training can improve control. Through miofeedback, the user might draw a better understanding of the requirements of the task and how to properly activate their muscles. The objective of this paper was to develop a miofeedback system that uses the electromyographic signal (EMG) to control a virtual prosthesis. The user receives visual feedback about their performance and the behavior of the EMG signal.
\end{abstract}

Keywords: miofeedback, myoelectric prostheses, EMG.

\section{Introdução}

O sinal EMG é resultado de uma ativação neuromuscular seguida pela contração muscular [1].
Esse tipo de sinal possui diferentes aplicações. Entre elas estão: determinar o tempo de contração muscular, a força produzida pelo músculo, o tempo que o músculo leva para fadigar e o comportamento geral das unidades motoras [2].

Graças às inúmeras informações sobre a coleta do sinal EMG e aos diversos campos de pesquisa na área, é possível criar tecnologias assistivas de diferentes tipos [3]. Nesse contexto estão inseridas as próteses mioelétricas, como uma forma de possibilitar maior independência de movimentação a pessoas com algum tipo de deficiencia motora.

As próteses mioelétricas funcionam de acordo com o sinal EMG do usuário, assim, a difculdade de controle do nível certo de ativação muscular ou a velocidade de resposta que o individuo possui são fatores fundamentais para a execução de diferentes tipos de tarefas [4].

O miofeedback é uma das diferentes modalidades de biofeedback existentes, juntamente com o neurofeedback, termofeedback, cardiofeedback e o biofeedback GSR (Galvanic Skin Response). Essas modalidades são, respectivamente, referentes à atividade cortical, à temperatura corporal, à frequência cardíaca e à resposta galvânica da pele. Por sua vez, o miofeedback é relativo à atividade muscular [5].

Graças às características do sinal EMG, o miofeedback é capaz de auxiliar no controle da atividade muscular, indicando a forma como a musculatura deve ser ativada para que a tarefa proposta seja realizada [6]. Desse modo, o miofeedback surge como uma ferramenta de orientação ao usuário, facilitando o processo de aprendizagem.

Uma das grandes vantagens do miofeedback consta na independência do indivíduo, já que ele recebe as informações suficientes para controle da prótese.

Dentro deste contexto, este artigo apresenta o desenvolvimento de um sistema de miofeedback em que o usuário é capaz de controlar uma prótese virtual a partir do sinal EMG dos músculos do antebraço.

\section{Materiais e métodos}

O sistema apresentado é constituído pelo hardware de captação do sinal EMG assim como pelo software que recebe esse sinal e mostra, em tempo real, a atividade elétrica do músculo em questão. 
Quando utilizado em terapias com miofeedback, o sinal EMG bruto pode ser ineficiente devido à sua natureza estocástica, sem uma forma de onda bem definida. Neste sentido, é comum que o sinal utilizado nos protocolos de treinamento seja a envoltória linear do sinal EMG que permite uma melhor visualização e delimitação da contração muscular [4]. Além disso, coletar apenas a envoltória do sinal EMG dimiui os requisitos do processo de digitalização, já que tanto o número de bits e a taxa de amostragem podem ser menores.

Para coletar a envoltória do sinal EMG, várias etapas são necessárias. Assim, o sinal EMG é coletado por eletrodos de superfície em configuração bipolar e o hardware deve ser responsável pela obtenção do envoltório linear. $\mathrm{O}$ diagrama de blocos do hardware desenvolvido é mostrado na Figura 1.

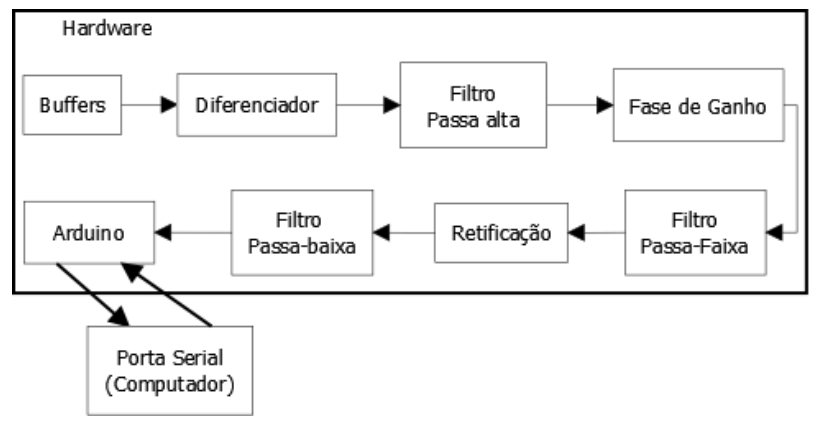

Figura 1: Diagrama de blocos do hardware utilizado contendo todas as etapas.

O sinal captado pelos eletrodos passa inicialmente por dois buffers e, em seguida, por um diferenciador a fim de eliminar os ruídos em modo comum, principalmente os relativos à rede $(60 \mathrm{~Hz})$. Após essa etapa, atua um filtro passivo com frequência de corte de $1 \mathrm{~Hz}$, eliminando sinais contínuos que venham a aparecer. O sinal deve ainda ser amplificado, com ganho de $361 \mathrm{~V} / \mathrm{V}$. O filtro passa-faixa é do tipo butterworth de segunda ordem e possui frequências de corte de 20 e $500 \mathrm{~Hz}$ e ganho de 1,56 V/V. O estágio de retificação também utiliza dois amplificadores operacionais constituindo um retificador de onda completa de precisão. O envoltório linear é obtido pelo filtro butterworth de segunda ordem passa-baixa com frequência de corte equivalente a $4 \mathrm{~Hz}$.

O sinal final é convertido de analógico para digital através do Arduino UNO. Além disso, a comunicação serial entre o Arduino e o computador permite que a transmissão de dados seja feita em tempo real.

A interface assim como o processamento do sinal foram implementados na linguagem de programação orientada à objetos $\mathrm{C \#}$. O formulário apresenta um teste de esforço com uma prótese virtual acionada pela contração muscular do usuário que a faz fechar.

Primeiramente, é feita uma coleta do sinal EMG em contração voluntária máxima (CVM). A partir deste valor, determina-se a porcentagem mínima da CVM necessária para fechar a prótese. Neste estudo, o limiar utilizado foi de $60 \%$. O objetivo do usuário é conseguir fechar a prótese e segurar um objeto que se desloca em sua direção no momento certo.

\section{Resultados}

Foram realizados testes para verificar o funcionamento do sistema. As figuras a seguir ilustram uma das sessões realizadas com um voluntário.

A Figura 2 mostra o circuito responsável pelo condicionamento do sinal EMG conectado ao Arduino UNO e à alimentação

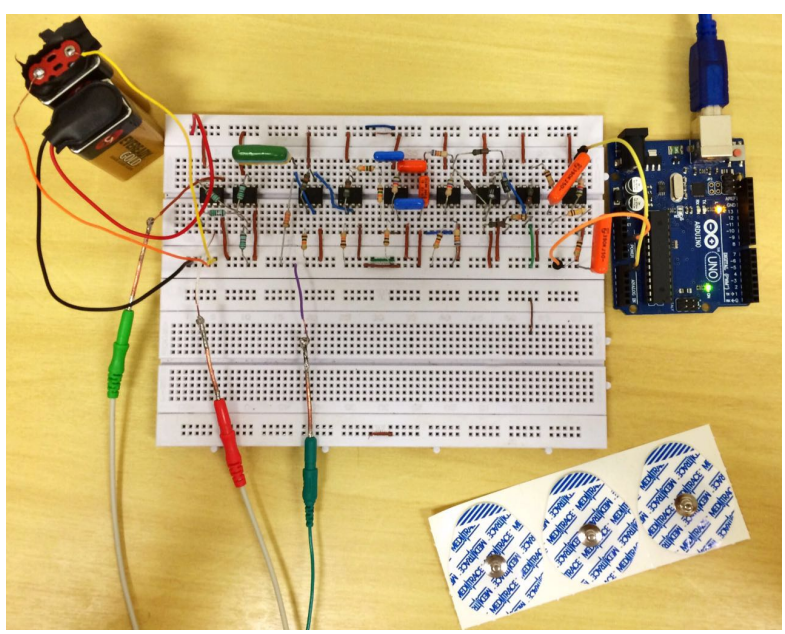

Figura 2: Hardware e eletrodos de superfície utilizados.

A Figura 3 mostra como foram posicionados os eletrodos no voluntário, sendo dois no músculo do antebraço para a coleta do sinal EMG e um eletrodo no ombro utilizado como referência.

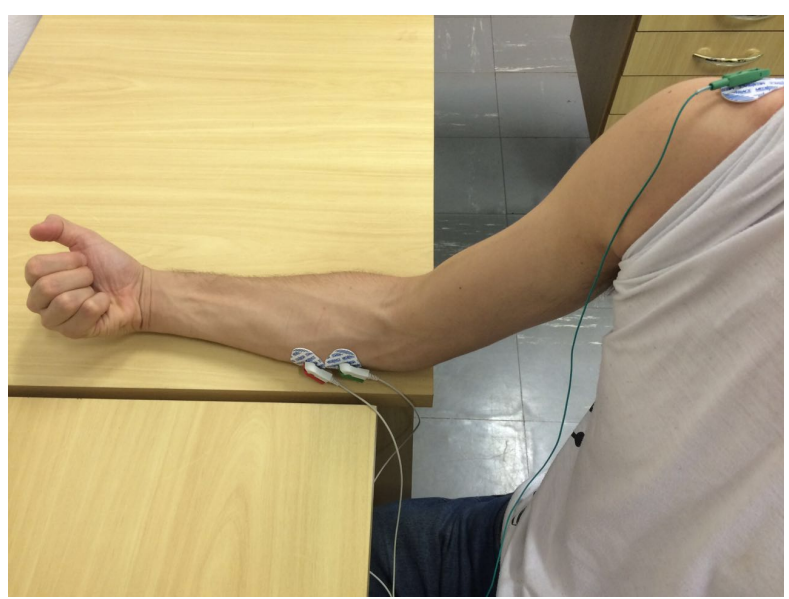

Figura 3: Posicionamento dos eletrodos para coleta do EMG no antebraço e eletrodo de referência no ombro do voluntário.

Após ser captado e amplificado, o sinal EMG aumenta sua amplitude sempre que o voluntário realiza atividade muscular, como mostra a Figura 4.

A Figura 5 apresenta graficamente a envoltória do sinal EMG do voluntário coletado pelo software 
desenvolvido, podendo assim classificar a força exercida.

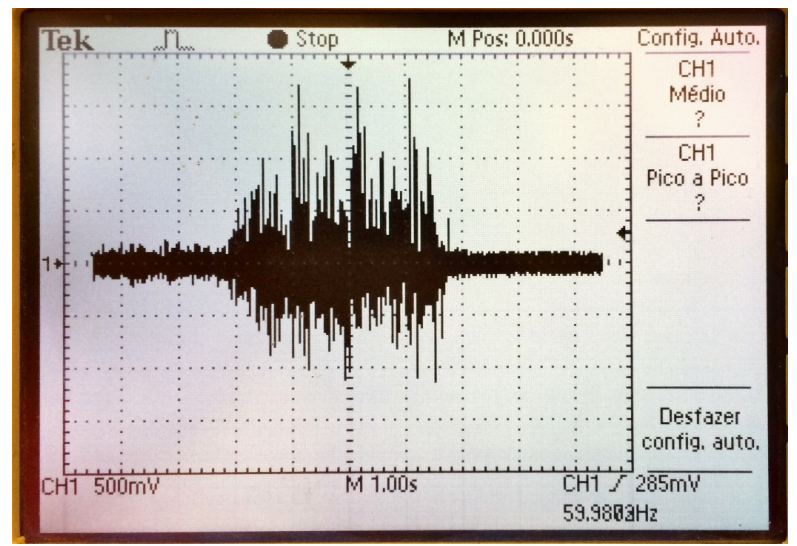

Figura 4: Sinal EMG amplificado.

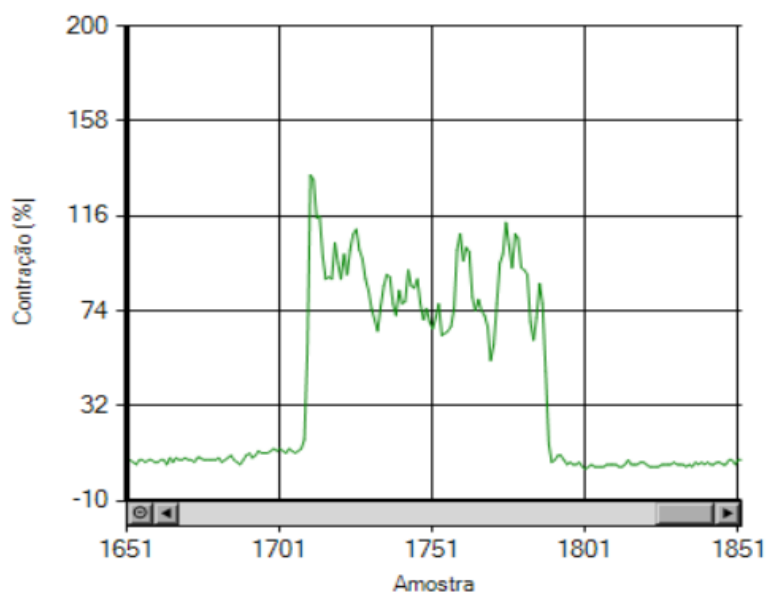

Figura 5: Envoltório linear do sinal EMG.

A figura 6 a mostra a prótese virtual em repouso, onde não há atividade muscular suficiente para fechála. Já na figura $6 \mathrm{~b}$ o usuário apresentou uma contração muscular suficiente para que a prótese se fechasse, sendo capaz de segurar o objeto no momento em que ele chega à posição alvo.

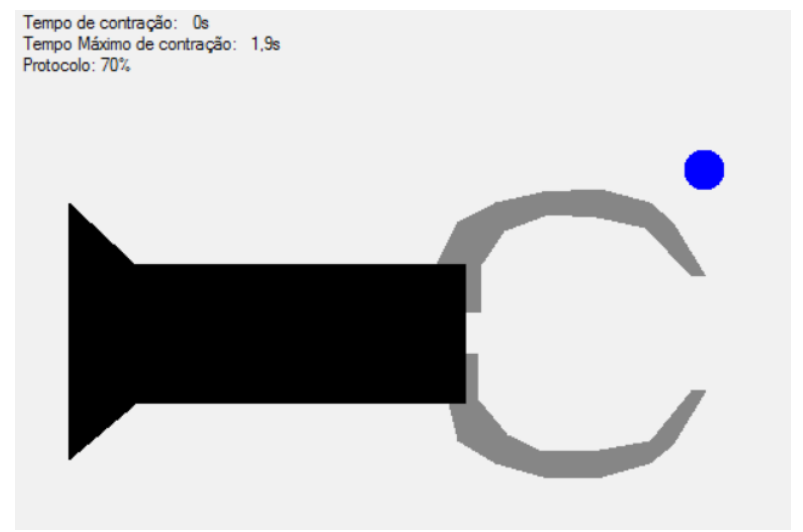

(a)

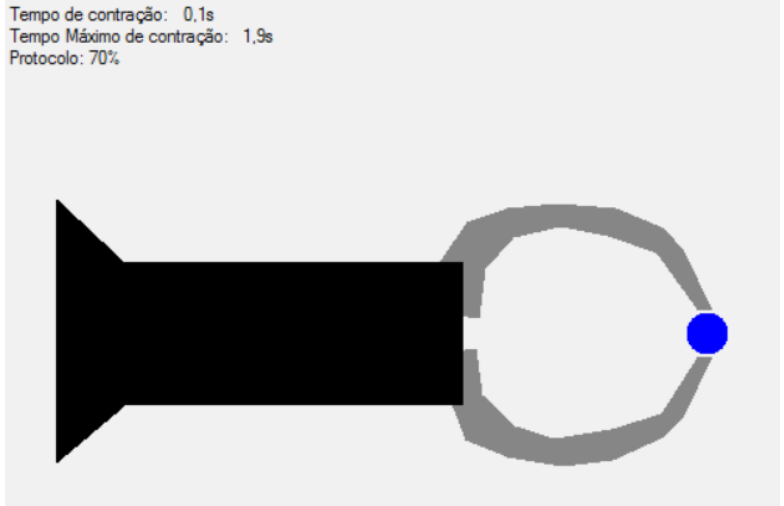

(b)

Figura 6: Prótese virtual criada via software. (a) Garras em repouso. (b) Garras fechadas.

\section{Discussão}

Os resultados obtidos mostram que o tempo de resposta do voluntário foi fundamental para que a tarefa fosse executada. Além disso, para que a bolinha permanecesse presa, era preciso manter o nível de contração muscular superior ao limiar. Logo, deve existir uma sincronia entre o tempo de resposta do usuário e a quantidade de tempo que ele consegue manter a musculatura ativa, respeitando o protocolo proposto.

No decorrer do estudo, foi possível analisar outras possíveis funcionalidades do miofeedback que também podem ser usadas como treinamento para uso de próteses. Nesse caso, a prótese se abre apenas se o indivíduo passa o limiar, entretanto, o miofeedback pode atuar, instruindo o usuário a controlar variáveis como a velocidade de abertura ou fechamento da garra [7]. Logo, o estudo do miofeedback pode ser usado para solucionar outros tipos de problemas sobre o controle de próteses mioelétricas como, por exemplo, a diferença na sensibilidade exigida para pegar um objeto considerado delicado, como um copo de vidro em relação à outro material mais rígido como, por exemplo um livro.

Nesse sistema, existe apenas um canal EMG. Entretanto, um sistema de hardware capaz de captar uma quantidade maior de canais também pode ser interessante, visto que garante mais graus de liberdade ao seu controle. Um artigo recente utilizou 64 canais EMG posicionados ao longo de todo o antebraço para decodificar movimentos individuais dos dedos [8], por exemplo.

Além disso, o tipo de movimento que aqui apresentado é bidimensional, pode ser desenvolvido em um sistema tridimensional com articulações que emulam o corpo humano, adicionando naturalidade ao movimento.

\section{Conclusão}

Trabalhos que envolvem miofeedback são interessantes para aprimorar o domínio de variáveis 
fundamentais para o controle de próteses, tais como a força e o tempo necessários.

Projetos futuros visam desenvolver o presente método envolvendo o aprimoramento do hardware de captação do sinal, adicionando mais canais EMG e outros tipos de variáveis de controle, incluindo próteses com maior grau de liberdade para movimentação, aumentado a dificuldade da tarefa. Além disso, o uso de outros tipos de biofeedback como o neurofeedback, podem ser implementados no processo de aprendizagem do usuário. Embora o sistema apresentado tenha como base uma prótese virtual, é preciso considerar o uso de próteses reais em estudos futuros, inserindo voluntários que necessitam desse tipo de treinamento para controle de próteses.

\section{Agradecimentos}

Agradecemos à Universidade Federal de Uberlândia e aos membros do laboratório de pesquisa do Biolab, pelo incentivo à pesquisa e pelo apoio dado durante o estudo.

\section{Referências}

[1]Guyton AC and Hall JE, Guyton and Hall Textbook of Medical Physiology, 11th ed. Elsevier Health Sciences, 2006.

[2] De Luca, G. Surface Electromyography: Detection aind Recording. Delsys; 2002.

[3] De Luca, G.Fundamental Concepts in EMG Signal Acquisition. Delsys; 2002.

[4] Chadwell A, Kenney L, Thies S, Galpin A and Head J. The reality of myoelectric prostheses: Understanding what makes these devices difficult for some users to control. Front. Neurorobot; 2016.

[5] Schwartz MS and Andrasik A. Biofeedback: A practioner's guide. 3rd ed. Guilford Press. 2005.

[6] Sá, AAR. Uma Proposta de Sistema Computacional em Tempo Real para Biofeedback. [Dissertação]. Uberlândia. Engenharia Elétrica: Universidade Federal de Uberlândia. 143p; 2004.

[7] Dosen S, Markovic M, Somer K, Graimann B, Farina D. EMG Biofeedback for online predictive control of grasping force in a myoelectric prosthesis. Journal of NeuroEngineering and Rehabilitation; 2012.

[8] Tenore FVG, Ramos A, Fahmy A, Acharya S, Etienne-Cummings R, and Thakor N V., Decoding of individuated finger movements using surface electromyography. IEEE Transactions on Biomedical Engineering. vol. 56, no. 5. pp. 1427-1434. 2009. 